\title{
Simulation of organic coating removal by particle impact
}

\author{
B. Zouari, M. Touratier* \\ LMSP-UMR CNRS-ENSAM-ESEM, 151 Boulevard de l'Hôpital, 75013 Paris, France
}

\begin{abstract}
The physical phenomena acting in paint removal by particle impact are not well known. Some simplified models assume that the paint removal is the result of erosion. Our research deals with the comprehension of the physical mechanisms which happen when an abrasive particle impacts a painted substrate. This paper addresses the numerical simulation of the impact of spherical particles on a substrate corresponding to an aluminum alloy sheet (A2024) coated with thermoset polyurethane paint (PAC 33). The behavior of the paint in the real conditions of decoating has been identified by solving an inverse problem using impact tests. Paint removal is the result of three phenomena: first, the initiation of delamination at the beginning of the impact due to major shear stresses at the interface; second, buckling of the paint film due to major radial compressive stresses in the film which come from the particle penetration in the coating; and third, the delamination of coatings by buckling occurs, according to the existing literature, in a combination of both mode I and II (mixed mode). The criterion of delamination is based on the state of stresses at the interface between the substrate and paint. This criterion has been introduced in finite element modeling. The numerical results are compared with experiments.
\end{abstract}

Keywords: Particle impact; Inverse problem; Finite element; Paint delamination

\section{Introduction}

The announcement of many restrictive rules and regulations concerning the protection of nature and the increasing use of composite parts in aircraft have encouraged the aeronautical industry to look for new processes for aircraft decoating. The industry is searching for solutions to substitute for the existing pollutant chemical processes without reducing the profitability nor the quality of surface decoating.

Stripping by media blasting pretends to be one such process. It consists of a stream of particles accelerated to a high velocity by compressed air and directed towards the coated substrate. The coating is, thus, removed by mechanical means. But there remains the problem of matching type of paint with the type of particle plus finding the optimal velocity and optimal impact angle to ensure maximum paint removal without damaging the substrate.

Many studies have been carried out on the analysis of contact between a rigid or elastic body on a layered solid. Diao et al. [1], and Bayashi and Yuan [2] have experimentally studied the fracture mechanisms of ceramic coatings in indentation. Komvapoulos [3] has used the finite element method to analyse the indentation of a layered solid in

\footnotetext{
* Corresponding author. Tel.: +33-1-44-246350; fax: +33-1-44-246468. E-mail addresses: bassem.zouari_ater@univ-orleans.fr (B. Zouari), maurice.touratier@paris.ensam.fr (M. Touratier).
}

normal contact with a rigid surface. Kral et al. [4,5] have studied the repeated indentation of an elastic plastic layered medium by a rigid sphere.

Papini and Spelt $[6,7]$ have studied organic coating removal by particle impact. They assumed that dynamic effects such as wave reflection at the interface are negligible. They found that coatings having high interfacial strength are removed by mechanical erosion [6], but coatings having weak interfacial strength are removed by delamination [7].

Delamination of a paint coating subject to compressive stresses, was studied by Evans and Hutchinson [8] and Yin [9]. Papini and Spelt [10] analyzed the buckling and delamination of a thin organic coating due to particle impact. All of these studies assumed that the coating has a linear behavior. In this paper, we present a finite element simulation of decoating of a paint layer, based on a model including large elastoplastic deformation at large strain rates. Specific impact experiments have been carried out in order to both characterize the paint behavior by solving an inverse problem, and to validate in detail the way in which the paint is removed under particle impacts.

\section{Experimental investigation}

An experimental test bench has been built [11] based on a gas gun used to accelerate spherical particles. The velocity of 


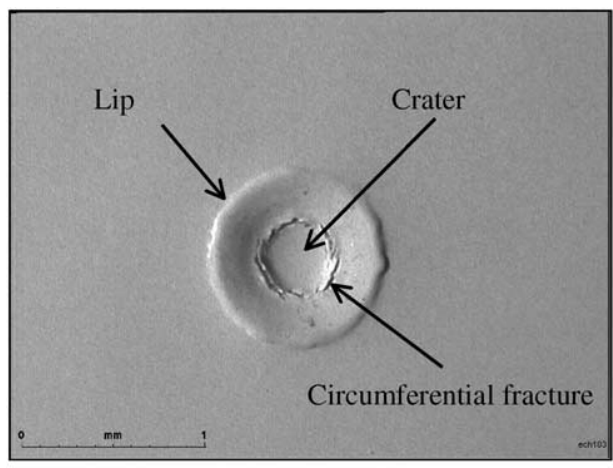

(a) $66 \mathrm{~m} / \mathrm{s}$

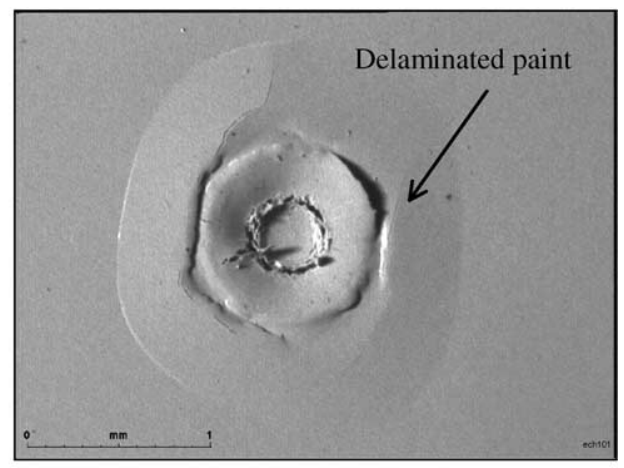

(b) $81 \mathrm{~m} / \mathrm{s}$

Fig. 1. Normal impacts with a $2 \mathrm{~mm}$ diameter alumina particle.

the particles is measured with an accuracy of $3 \%$ by timing their flight between two infrared light beams located near the muzzle of the gun.

Three different materials were used as particles: steel 100C6, alumina and polyacetal. In this paper, we present only the result of the impact with alumina particles. In fact, with steel particles, the phenomena are similar to those observed for alumina. On the other hand, polyacetal particles did not result in any imprint or erosion mechanisms, even at high velocity $(200 \mathrm{~m} / \mathrm{s})$. Specimens used were aluminum alloy circular plates $30 \mathrm{~mm}$ in diameter and $1 \mathrm{~mm}$ thick, coated with a $0.1 \mathrm{~mm}$ polyurethane paint layer, and clamped on their circumference.

A parametric study has been conducted according to variations of impact speed and angle.

\subsection{Normal impact}

When the particle speed is high enough to create paint delamination, a central region (of disk shape, see Fig. 1) of the imprint remains painted. This phenomenon is due to excessive plastic strains in the paint under the particle during impacts which, thus, promotes the adhesion of the paint. The radius of the cracked region is independent of the particle velocity. It varies with particle diameter. Fig. 1a shows a $66 \mathrm{~m} / \mathrm{s}$ velocity impact site on the paint at normal incidence. The coating did not delaminate, but the impact created an indentation and there is plastic paint deformation and a circumferential cracking pattern. Removal of the paint layer in the impact region revealed plastic deformation in the substrate.

When the velocity increases, the level of paint damage increases and for an impact velocity of $81 \mathrm{~m} / \mathrm{s}$, we find that there are two regions, see Fig. 1b. The first is the center of the imprint where the paint is still present, while the second is that where the paint is delaminated. The radius of this last region is about $1 \mathrm{~mm}$, the diameter of the particle being $2 \mathrm{~mm}$.

\subsection{Oblique impact}

Fig. 2a and $b$ present the imprint of oblique impact with an initial velocity equal to $60 \mathrm{~m} / \mathrm{s}$ and for impact angles equal to 60 and $30^{\circ}$, respectively.

For oblique impacts, it is seen that paint delamination occurs at an impact velocity lower than in a normal impact. Fig. 2a shows the presence of circumferential cracks. The

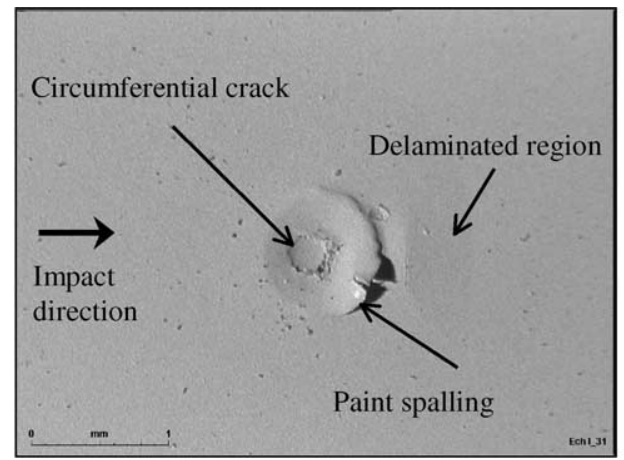

(a) $60^{\circ}$

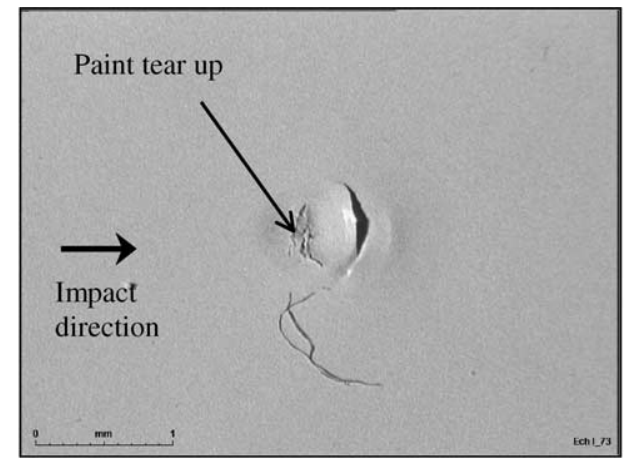

(b) $30^{\circ}$

Fig. 2. Oblique impacts at $60 \mathrm{~m} / \mathrm{s}$ velocity ( $2 \mathrm{~mm}$ particle diameter). 
delamination region is localized in the opposite direction of the incoming particle direction. In $30^{\circ}$ oblique impact, the paint does not delaminate but we notice a ripping of the paint.

To try to understand the main mechanism responsible for paint delamination, we simulate impact problems corresponding to the above tests, by using a finite element software.

\section{Mechanical characterization of the paint under impact}

To simulate the impact of a spherical particle on a coated substrate, we need to characterize, under impact, the mechanical behavior of the coating film. This task is difficult, especially because the paint thickness is only about $100 \mu \mathrm{m}$ and it is subject to high strain rates.

In a classic traction test on a paint film, only a crack in the film was seen, but no plastic deformation [11]. This is due to the difference in strain rates acting in the traction (about $10^{-2} \mathrm{~s}^{-1}$ ), and impact (about $10^{5} \mathrm{~s}^{-1}$ ) tests, which leads to two different deformation mechanisms. In the first case, we have essentially viscoelastic strains, while in the second we have plastic strains.

The constitutive law of the paint must take into account the effect of strain rates. In order to include sensitivity to the above phenomena, the flow stress of the paint is modeled by the Johnson-Cook law as follows [12]:

$\bar{\sigma}=\left(A+B \bar{\varepsilon}^{n}\right)\left(1+C \ln \dot{\bar{\varepsilon}}^{*}\right)\left(1-T^{* m}\right)$

where $\bar{\varepsilon}$ is the equivalent plastic strain, $\dot{\bar{\varepsilon}}^{*}=\dot{\bar{\varepsilon}} / \dot{\varepsilon}_{0}$ the dimensionless plastic strain rate for $\dot{\varepsilon}_{0}=1 \mathrm{~s}^{-1}, T^{*}=(T-$ $\left.T_{\text {room }}\right) /\left(T_{\text {melt }}-T_{\text {room }}\right)$ the homogenous temperature, $T_{\text {room }}$ the room temperature, $T_{\text {melt }}$ the melt temperature, $A$ the elastic limit of the material, $B$ and $n$ the hardening coefficients, $C$ the viscosity, and $m$ the thermal softening coefficients which is neglected in our study.

To obtain data for the Johnson-Cook law coefficients of the paint, within impact loading conditions, we have to solve an inverse problem [13-16]. The aim of this technique is to compare the impact crater dimensions issued from experiments with those coming from finite element computations achieved as stated hereafter in Section 4. Thus, the inverse problem is given as a parameter estimation problem where the four unknowns are $A, B, n$ and $C$.

The researched unknowns $A, B, n$ and $C$ are those which minimize in the least square sense the difference $\Phi$ between experimental and computational dimensions of the crater. This is accomplished by minimizing the error function $\Phi$, with respect to the set $m$ of the parameters.

A crater in a paint layer is characterized by its depth $(p)$, diameter $(d)$ and lip height $(h)$, see Fig. 3. The experimental dimensions used in our objective function are those issued from the following two normal impact tests. In the first, the particle is of alumina and has a $2 \mathrm{~mm}$ diameter and an initial

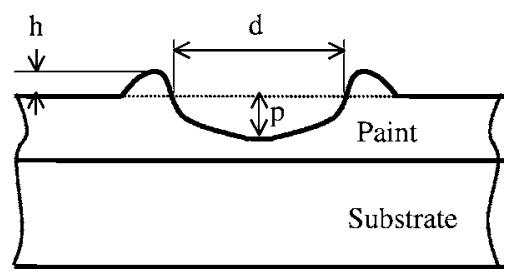

Fig. 3. Crater dimensions.

velocity of $45 \mathrm{~m} / \mathrm{s}$. In the second one, the particle is of steel $100 \mathrm{C} 6$ and has a $2 \mathrm{~mm}$ diameter and an initial velocity of $35 \mathrm{~m} / \mathrm{s}$.

The error function to be minimized is then defined by:

$\Phi(m)=\sum_{i=1}^{2}\left[\left(1-\frac{h_{i}}{\hat{h}_{i}}\right)^{2}+\left(1-\frac{p_{i}}{\hat{p}_{i}}\right)^{2}+\left(1-\frac{d_{i}}{\hat{d}_{i}}\right)^{2}\right]$

where $\hat{\alpha}_{i}$ are experimental crater dimensions issued from the $i$ th measure, and $\alpha_{i}$ are crater dimensions deduced from finite element simulations of the corresponding impact.

Because the finite element model is non-linear with respect to the parameters, the minimization is referred to as a non-linear least square problem.

The minimization of Eq. (2) is subjected to constraints on the unknowns $A, B, n$ and $C$ based on the physics of the problem. These constraints are written in the form:

$C_{j}(m) \geq 0$

The constraints in our case must deal with the thermal activation theory developed by Eyring [17]. They result in [18]:

$\left\{\begin{array}{l}A-0.1 \geq 0 \\ 2-A \geq 0 \\ C-0.012 \geq 0 \\ 0.12-C \geq 0 \\ B \geq 0 \\ n \geq 0\end{array}\right.$

The constraints are enforced by the interior penalty function method, which is an unconstrained sequential minimization technique, and they are incorporated directly in the penalized multi-criteria objective function $\Phi^{*}$, as follows:

$\Phi^{*}(m)=\Phi(m)+\sum_{j=1}^{q} \zeta_{j}(m)$

where the weighted penalty functions $\zeta_{j}$, are the inverse barrier functions proposed by Carrol [19], and are expressed by:

$\zeta_{j}(m)=\frac{\omega_{j}}{C_{j}(m)}$

in which the weighting coefficients $\omega_{j}$ are to be adjusted. The advantage of the penalty function method is that the constrained problem is solved as unconstrained through the 


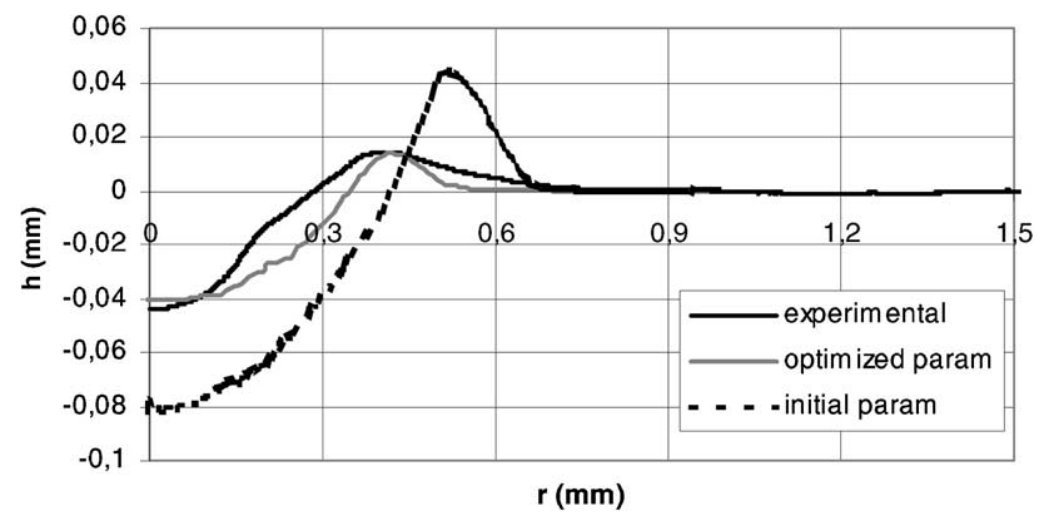

Fig. 4. Crater shape before and after optimization.

minimization of Eq. (5). To minimize the objective function we used the modified Levenberg-Marquardt method [20,21].

As a result, Fig. 4 shows an experimental crater shape and two calculated craters using either arbitrary or optimized parameters, corresponding to a $35 \mathrm{~m} / \mathrm{s}$ normal impact velocity with a steel particle. Note that similar results regarding the predicted crater shape, have been obtained in the case of a velocity of $45 \mathrm{~m} / \mathrm{s}$ and an alumina particle. The values of the optimized parameters are $A=1.46 \times 10^{-1} \mathrm{GPa}, B=$ $0.15 \mathrm{GPa}, C=0.097$ and $n=0.498$.

Finite element computations to achieve the mentioned inverse problem have been deduced from an elastoplastic model taking into account large deformation and large strain rates as explained now.

\section{The finite element model to simulate paint removal from particle impact}

In this section, we model and simulate the impact of a spherical particle on an aluminum alloy plate (A2024) coated with a polyurethane paint (PAC33). The thickness of the plate and paint are, respectively, 1 and $0.1 \mathrm{~mm}$. The plate is circular with a radius of $15 \mathrm{~mm}$. The plate is assumed clamped on its circumference. Both paint and sub- strate have elastoplastic behavior. The paint flow limit follows the Johnson-Cook law as mentioned in the previous section. The substrate is assumed to have a perfect elastoplastic behavior with a plastic flow limit equal to $465 \mathrm{MPa}$. The Young's modulus of the substrate is equal to $71 \mathrm{GPa}$. The Young's modulus of the paint is equal to $2.3 \mathrm{GPa}$. It has been obtained from traction tests on paint films at several strain rates $\left(10^{-4}\right.$ to $\left.10^{-2} \mathrm{~s}^{-1}\right)$. Prony's series have been introduced to deduce the relaxation function [22].

This problem is quite complex, with large strains, high strain rates, plastic constitutive law and contacts. In our numerical simulation, we used a dynamic explicit finite element software (LS-DYNA).

In a non-linear analysis, the equilibrium of the body considered must be established in the current configuration, which is unknown. In general, it is necessary to employ an incremental formulation and to use a time variable to consistently describe the loading and the motion of the body [23].

To solve the equation of motion, we used the finite element method for the space discretization. Our problem has a symmetric plane, so we only consider half of both the particle and plate. The region under the impact (around three times the diameter of the particles) is meshed with three-dimensional eight node elements (HEXA8), see Fig. 5, as the impact is very localized, while the rest of the plate is

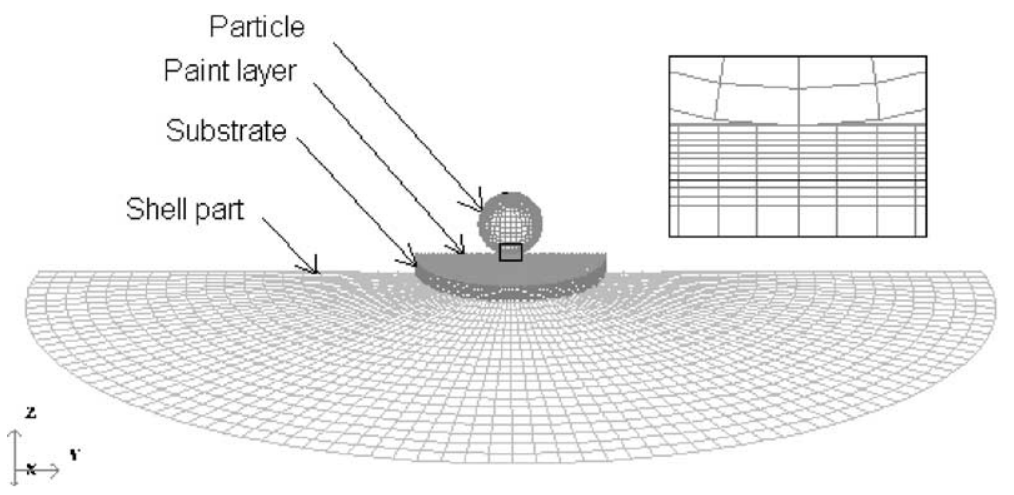

Fig. 5. The mesh used. 


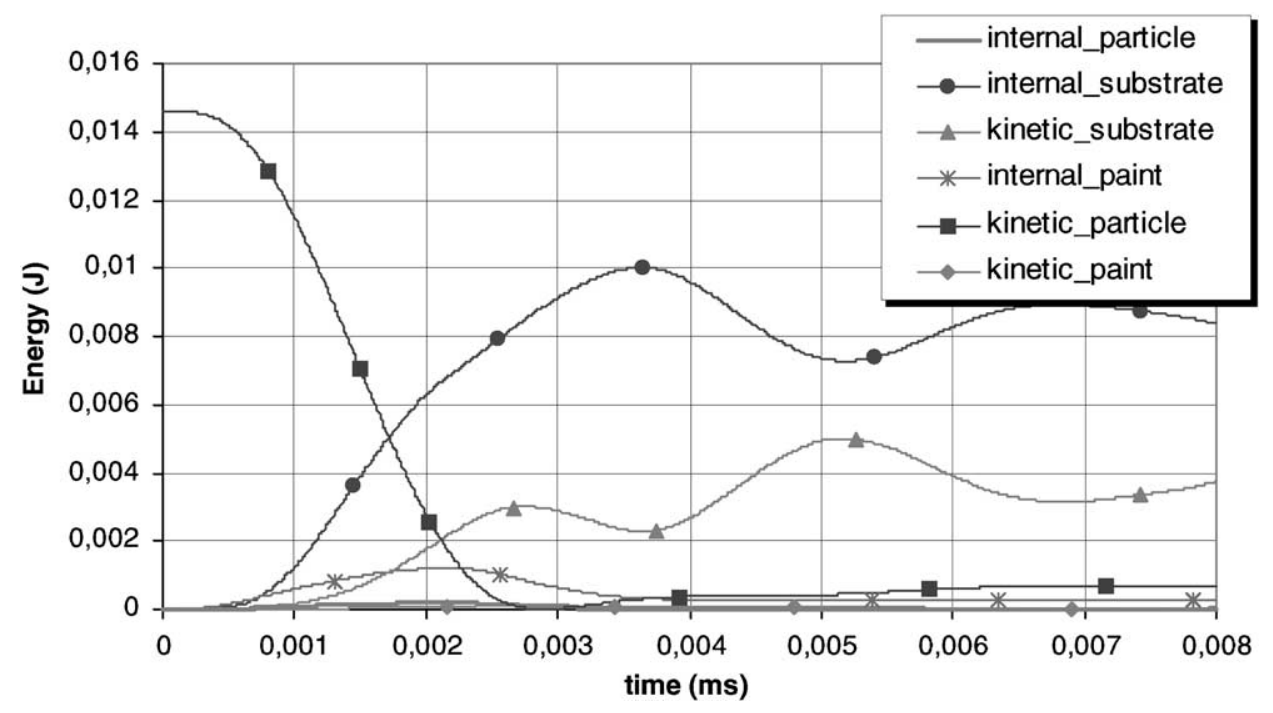

Fig. 6. Internal and kinetic energy evolution in the case of a normal impact.

meshed with four node Belytchko shell elements [24]. The junction between the two types of mesh is achieved by introducing kinematic conditions. During the calculation, brick nodes located at the junction surface are constrained to lie along the full fiber thickness of the shell, having the capability to move relative to each other in this fiber direction.

The target elements have a full integration scheme to avoid possible hourglass modes during simulations. The other elements have a reduced integration scheme. In our model, there are 46,000 brick elements and 1600 shell elements, giving 206,514 degrees of freedom.

\section{Results}

Using the previous model, we have simulated the impact of alumina spherical particles on an aluminum plate coated with a polyurethane paint in conditions similar to the experiments. In a first case we assumed that the interface between the coating and the substrate was perfect.

\subsection{Energetic balance-sheet}

Although the great difference between the Young's modulus of the paint $(2.3 \mathrm{GPa})$ and that of the particles $(380 \mathrm{GPa}$ for alumina), we consider that the particles are elastic, contrary to the major part of the studies [1,2] which take the particles as rigid. In fact, during impact the particle is subjected to a very high stress level which induces its deformation.

Energetic distributions have been analyzed in all parts of our mechanical system (particle, substrate and paint) for two cases of impacts: normal and oblique. Figs. 6 and 7 represent the evolution of internal and kinetic energies in the

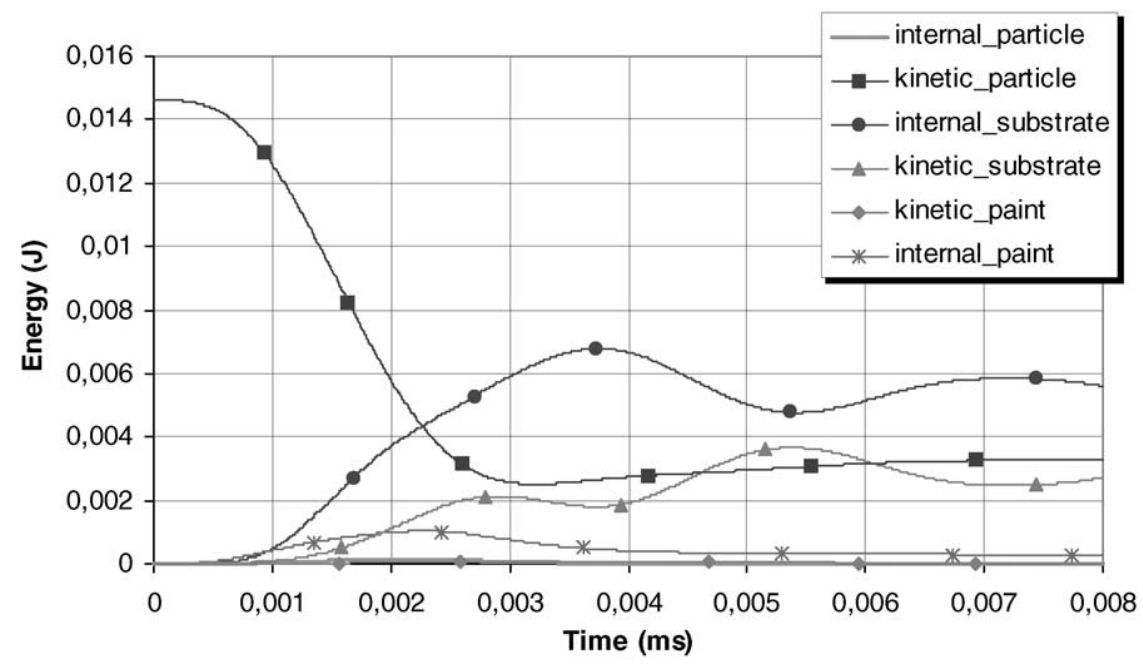

Fig. 7. Internal and kinetic energy evolution in the case of an oblique impact at $60^{\circ}$. 


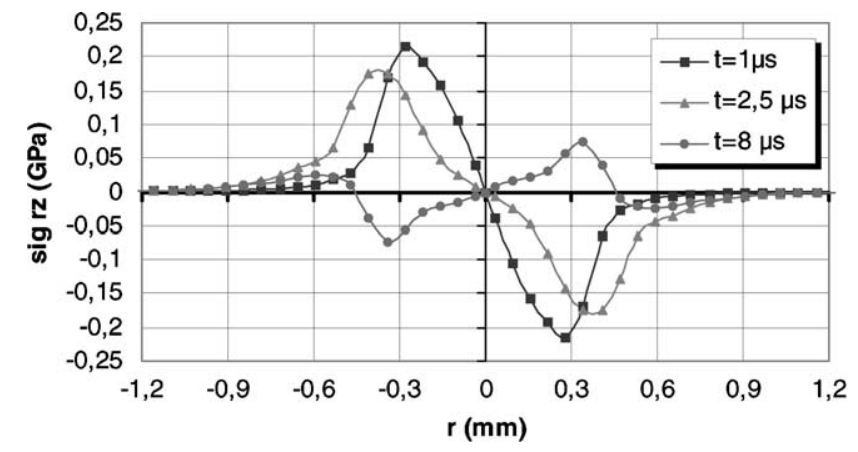

Fig. 8. Variation of interface shear stresses with the radial distance for different instances of normal impact.

substrate, coating and particle, respectively, for the normal and oblique (under an angle of $60^{\circ}$ ) impacts.

We notice that the substrate consumes a large amount of energy which is divided into two parts: the first is kinetic energy and the second is the internal one. The substrate internal energy is greater in the case of normal impacts than in oblique impacts. We can conclude that the substrate has a greater degree of plastic deformation and damage in normal impact than in an oblique one. This result is confirmed by the fact that the kinetic energy of particle rebound is greater in an oblique case than in a normal case.

\subsection{Stress state}

The study of the state of stresses at the interface is required to understand the mechanisms acting in the paint removal by particle impacts. Papini and Spelt $[7,10]$ assumed that shear stresses at the interface are mainly responsible for coating removal in the weak interfacial strength coating case. We have studied the variation of shear stresses along the interface between paint layer and substrate, with the radial distance from the center of the impacted region at different impact times. Figs. 8 and 9 shown these evolutions, respectively, in the case of normal and oblique impacts by an alumina particle under an initial velocity of $60 \mathrm{~m} / \mathrm{s}$.
These two cases of impact show that the shear stresses reach their maximum $1 \mu \mathrm{s}$ after the beginning of the impact. These maxima are located at $0.26 \mathrm{~mm}$ from the center of impacted region if the impact is normal, and at $0.35 \mathrm{~mm}$ for the oblique case. These stresses are responsible for the circumferential cracks observed during experiments (Figs. 1 and 2). In oblique impacts, shear stresses are greater in front of the particle (in the right region of Fig. 9) than behind it.

Shear stresses induce the initiation of paint delamination but they are not alone responsible for paint removal because they become negligible at a radius distance equal to $0.6 \mathrm{~mm}$ for a normal impact, and at $0.8 \mathrm{~mm}$ for an oblique one. In addition, their effect is very localized under the region of impact.

To have a more realistic finite element model, we must take into account the separation of the paint from the substrate.

\section{Paint removal model}

Delamination and sapling of coatings subjected to compressive stresses have been studied by many authors. Evans et al. [8] have developed a model based on the fracture

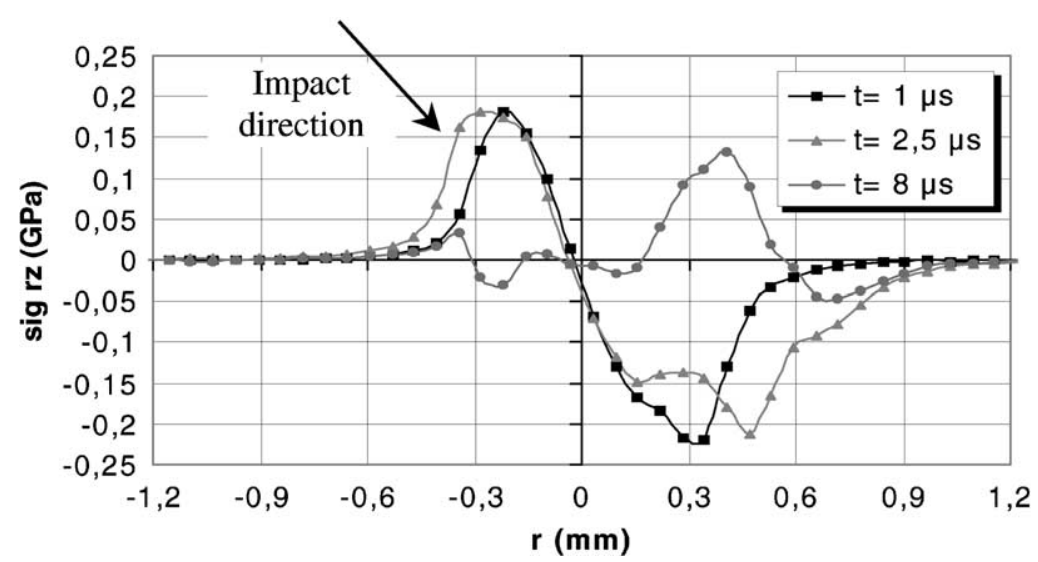

Fig. 9. Variation of interface shear stresses with the radial distance for different instances of oblique impact. 

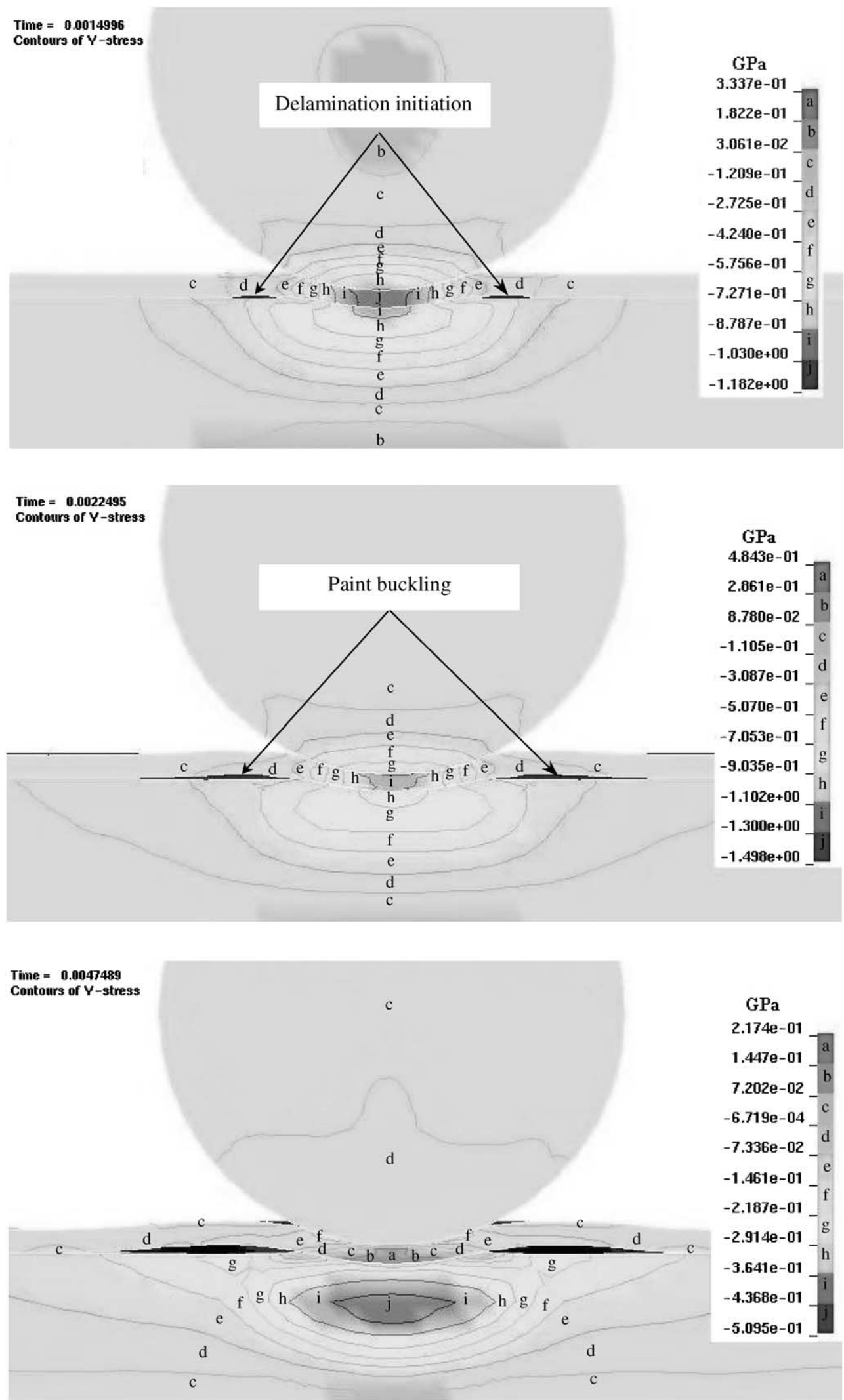

Fig. 10. Paint delamination evolution in the case of normal impact $(V=81 \mathrm{~m} / \mathrm{s})$. 

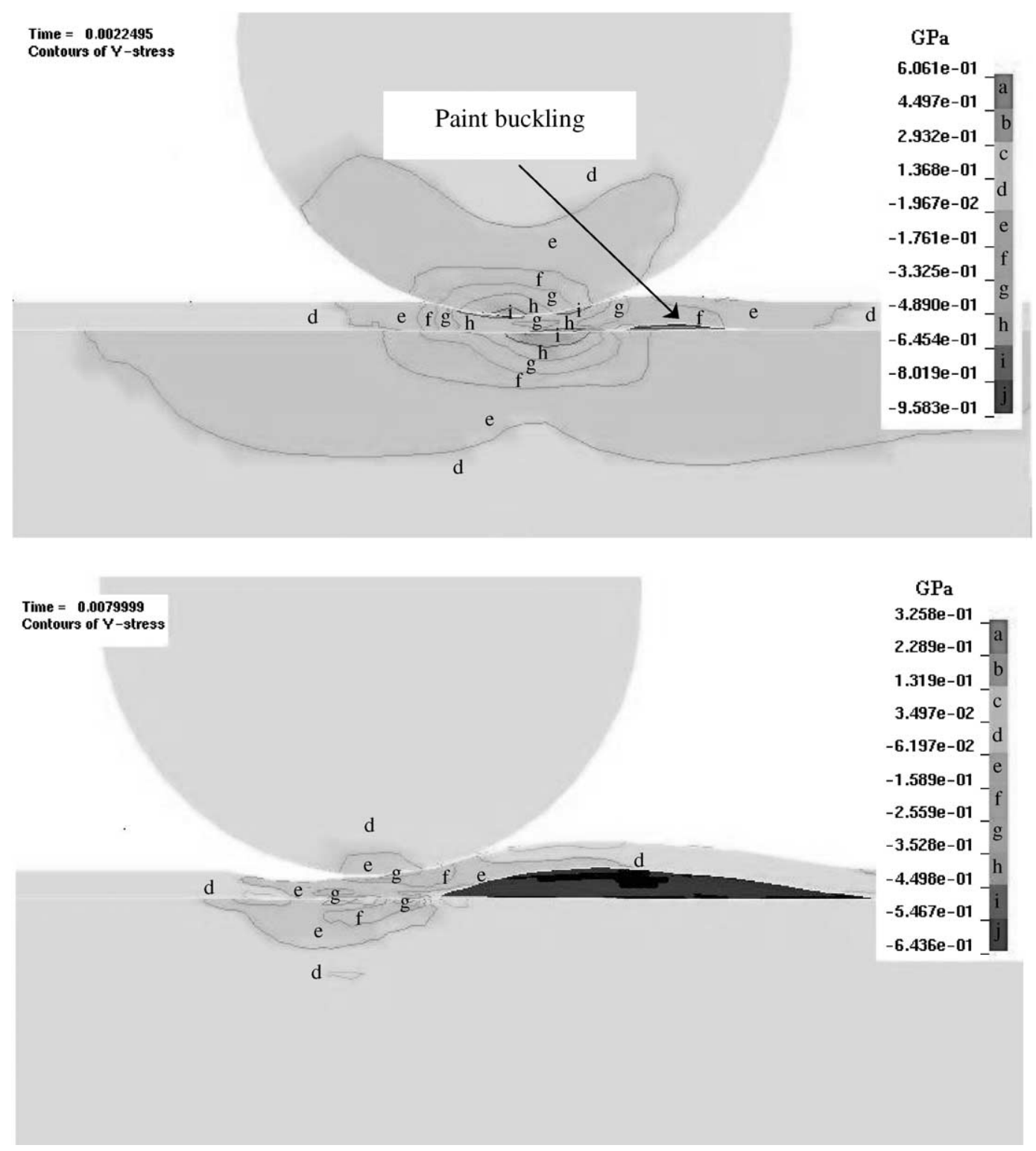

Fig. 11. Paint delamination evolution in the case of oblique impact at $60^{\circ}(V=60 \mathrm{~m} / \mathrm{s})$.

mechanics theory and the plate buckling theory. The authors demonstrate that the delamination of pre-compressed films occurs if the film buckles, while a stress concentration appears at the buckle perimeter. Yin [9] has used an iterative procedure based on the fourth-order Runge-Kutta integration formula, to generate a family of non-dimensionalized postbuckling solutions of the von Karman's non-linear plate theory. These solutions determine the change of the energy-release rate with the growth of a circular thin film or midplane delamination, which in turn determines the stability characteristics of growth.

When a coating is subjected to an indentation, the corresponding penetration into the coating induces compressive stresses. These stresses, in the presence of the initial delaminated region of the coating can induce buckling of the coating and then the propagation of delamination [10]. Papini and Spelt [10] developed a model for predicting the coating buckling under normal impact by a spherical particle. They assumed that the region of the coating in contact with the particle can not buckle.

In order to study the propagation of paint delamination under particle impacts, we have introduced a debonding criterion between paint and substrate in our finite element model. We adopt a special finite element mesh. Initially, corresponding nodes on the substrate surface and on the paint surface are perfectly bonded, and therefore, have identical displacements. During the simulation, the nodes debond introducing tear-off of the paint layer. This node separation is governed by the following debonding criterion:

$$
\left(\frac{\left\langle\sigma_{\mathrm{n}}\right\rangle}{\mathrm{NFLS}}\right)^{2}+\left(\frac{\left|\sigma_{\mathrm{s}}\right|}{\mathrm{SFLS}}\right)^{2} \leq 1
$$


where $\left\langle\sigma_{\mathrm{n}}\right\rangle$ is the positive part of the stress vector normal to the interface, $\sigma_{\mathrm{s}}$ is the shear stress, NFLS and SFLS are, respectively, normal and shear failure stresses. Failure is assumed if the left hand side of (7) is greater than one.

The values of NFLS and SFLS are deduced from tear-off tests [11] and they are, respectively, equal to 50 and $200 \mathrm{MPa}$. This criterion does not take into account the results of the fracture mechanics theory.

We have applied this model for a $81 \mathrm{~m} / \mathrm{s}$ velocity normal impact (Fig. 10) with a $2 \mathrm{~mm}$ diameter alumina particle and for an oblique impact at $60^{\circ}$ with the same type of particle and with an initial velocity equal to $60 \mathrm{~m} / \mathrm{s}$ (Fig. 11). As observed from experiments, see Figs. $1 \mathrm{~b}$ and $2 \mathrm{a}$, the present numerical model predicts that delamination does not occur under the particle. This is due to this region being subjected to high normal compressive stresses. Therefore, the debonding criterion given by Eq. (7) is not reached.

Fig. 10 shows the evolution of radial stresses in the structure at different normal impact times. At the beginning of the impact, the paint delamination is initiated. When radial compressive stresses are enough to induce the paint buckling, the paint is buckled and then the delamination propagates till the particle rebounds.

Fig. 11 shows the evolution of radial stresses in the paint and substrate at different times for oblique impacts. The paint delamination is initiated around the limit of the contact region between the particle and paint but the delamination propagates only from the side in front of the particle. In fact, in this region the radial stresses in the paint are in compression and higher than in the region behind the particle. The paint buckling then occurs only in a region in front of the particle.

We have compared the dimensions of the delaminated region issued from measurements using micrographies (from an optical microscope) and numerical simulations. We found that in normal impact the diameter of the delaminated region after experiments (repeated five times) was equal to $2 \mathrm{~mm}$ and based on simulations was equal to $2.3 \mathrm{~mm}$, which is near the experimental value. However, in oblique impacts we noticed that the delaminated region is overestimated relative to our model.

\section{Conclusion}

The mechanical simulation of the impact is a good tool for understanding the paint removal mechanism. It allows us to analyze the stress state at different stages of the impact. We note that the most difficult part is finding numerical data for the paint constitutive law. For this reason, an inverse method has been carried out to find the coefficients of the Johnson-Cook law of the paint.

Paint delamination is initiated by shear stresses at the beginning of impact. When the particle penetrates inside the coating, the radial stresses are compressive and induce paint buckling when they reach a critical value. Paint delamination propagates following a mixed mode.

Finally, to extend the present work to the more practical problem of decoating of paint layers by using media blasting, a stochastic multi-impact model of particles has to be developed.

\section{References}

[1] D. F Diao, K. Kato, K. Hokkirigawa, Fracture mechanisms of ceramic coatings in indentation, ASME J. Tribol. 116 (1994) 860-869.

[2] K. Bayashi, F. Yuan, Elastic-plastic analysis of stresses and initiation of cracks in a ceramic coating under indentation by an elastic sphere, ASME J. Tribol. 120 (1998) 463-469.

[3] K. Komvopoulos, Finite element analysis of a layered elastic solid in normal contact with a rigid surface, ASME J. Tribol. 110 (1998) 477-485.

[4] F.R. Kral, K. Komvopoulos, D.B. Bogy, Finite element analysis of repeated indentation of an elastic-plastic layered medium by a rigid sphere. Part I. Surface results, ASME J. Appl. Mech. 62 (1995) 20-28.

[5] F.R. Kral, K. Komvopoulos, D.B. Bogy, Finite element analysis of repeated indentation of an elastic-plastic layered medium by a rigid sphere. Part II. Subsurface results, ASME J. Appl. Mech. 62 (1995) $29-42$.

[6] M. Papini, J.K. Spelt, The plowing erosion of organic coatings by spherical particles, Wear 222 (1998) 38-48.

[7] M. Papini, J.K. Spelt, Organic coating removal by particle impact, Wear 213 (1997) 185-199.

[8] A.G. Evans, J.W. Hutchinson, On the mechanics of delamination and spelling in compressed film, Int. J. Solid Struct. 20 (5) (1984) 455-466.

[9] W.L. Yin, Axisymmetric buckling and growth of a circular delamination in a compressed laminate, Int. J. Solid Struct. 21 (5) (1985) 503-514.

[10] M. Papini, J.K. Spelt, Indentation induced buckling of organic coatings. Part I. Theory and analysis, Int. J. Mech. Sci. 40 (10) (1998) 1043-1059.

[11] B. Zouari, Modélisation et Simulation de l'Enlèvement de Revêtements Polymériques par Impact Particulaire, Thèse de doctorat, No. 2001-10, Ecole Nationale Supérieure d'Arts et Métiers-CER, Paris, June 2001.

[12] G.R. Johnson, W.H. Cook, A constitutive model and data for metals subjected to large strains, high strain rates and high temperatures, in: Proceedings of the Seventh International Symposium on Ballistics, The Hague, 1983, pp. 541-547.

[13] H.D. Bui, Introduction aux problèmes inverses en mécanique des matériaux Eyrolles, 1993.

[14] M. Bonnet, Le problème mathématique de l'inversion: généralités, solutions exactes, in: Problèmes Inverses de L'expérimentation à la Modélisation, OFTA, Paris, October 1999, pp. 41-58.

[15] E. Massoni, Identification automatique du comportement par analyse inverse, Séminaire de Plasticité et Mise en Forme des Métaux, CEMEF, Ecole des Mines de Paris, Paris, 1994 (Chapter 20).

[16] A. Tarantola, Inverse Problem Theory, Elsevier, Amsterdam, 1987.

[17] M. Brown, Yield behavior of polymers, in: W. Brostow, R.D. Corneliussen (Eds.), Failure of Plastics, Hanser, Munich, 1986, Chapter 6, pp. 98-118.

[18] D.W. Van Krevelen, Thermophysical properties of polymers, in: Properties of Polymers, Elsevier, Amsterdam, 1990, pp. 71-286.

[19] C.W. Carroll, The created response surface technique for optimizing non-linear restrained systems, Operat. Res. 9 (1961) 169-184. 
[20] D.W. Marquardt, An algorithm for last-squares estimation of nonlinear parameters, J. Soc. Ind. Appl. Math. 11 (2) (1963) 431441.

[21] D.S. Schnur, N. Zabaras, An inverse method for determining elastic material properties and a material interface, Int. J. Num. Methods Eng. 33 (1992) 2039-2057.

[22] B. Zouari, M. Touratier, Numerical simulation of aeronautical paint layer decoating, in: B.H.V. Topping (Ed.), Proceedings of the Fifth
International Conference on Computational Structures Technology, Leuven, Belgium, 6-8 September 2000, Finite Elements: Techniques and Developments, Edinburgh, UK: Civil-Comp Press, pp. 231-236.

[23] K.J. Bathe, Finite element nonlinear analysis in solid and structural mechanics, Finite Element Procedures, Prentice-Hall, Englewood Cliffs, NJ, 1996, pp. 485-641.

[24] J.O. Hallquist, LS-DYNA3D Theoretical Manual, Livermore Software Technology, 1998. 